\title{
Applications of a Bayesian Based Maximum Entropy Method to Energy Dispersive X-ray Spectra
}

\author{
T. Shinkawa*, N. Nakamura*, H. Kato*, K. Shibuya** and M. Nakata*** \\ *JEOL Ltd., 3-1-2, Musashino, Akishima, Tokyo 196-8558 Japan \\ **JEOL ENGINEERING CO., Ltd., the same address as JEOL \\ *** Tokyo Univ. of A \& T, 2-24-16, Naka-cho, Koganei, Tokyo 184-8558 Japan
}

An energy dispersive X-ray detector is widely used for chemical analysis to measure intensity spectra of characteristic X-rays of component elements in an analyzing specimen. From the peaks of a measured spectrum, the weight percentages of each element are determined. In a long-time measurement to obtain a low noise spectrum with the increase in probe current, the spatial resolution of X-rays decreases partly due to the radiation of X-rays from the surrounding area larger than the probe size itself, and partly due to the drifts of the electron beam and/or of the specimen stage. In a short-time measurement to avoid the above difficulties, the X-ray spectrum contains a much higher level of noise. The intensity of the X-ray becomes much weaker because the radius of the electron beam is about several microns and the beam current is of the order of several nano-amperes. It has been well known that usual smoothing techniques downgrade the sharpness. Up to the present there has been no useful method reported to decrease the noise without degrading the sharpness of the spectrum. In the present study, a Bayesian based maximum entropy method (BBMEM) is applied to the spectrum obtained for a few seconds, and is proved to be one of the most effective techniques to reduce the noise in the spectrum.

BBMEM is derived from both Bayes' theorem, which provides inductive inference in scientific phenomenon, and the latest Maximum Entropy Method [1]. This method is widely used for image reconstructions in radio astronomy and molecular biology and for spectral analyses in various fields [2] [3]. It is a powerful technique to infer the most suitable figure from an observed weak signal with high noise.

We have applied BBMEM to an actual EDX spectrum of a specimen that contains rare metal of Nd. The sampling time is $5 \mathrm{~s}$. As shown in Fig. 1a, the original spectrum contains a high level of noise. The improved spectrum (Fig.1 b) processed by BBMEM, in which the curves seem to become smoother, maintains the sharpness of each peak. Figure $1 \mathrm{c}$ ) is a superimposed spectrum of the original one with artificial random noise, while Fig.1 d) is the improved spectrum of Fig.1 c) processed by BBMEM. The spectrum of Fig.1 d) shows an effective reconstruction of the original spectrum of Fig.1a). The 
present result demonstrates that BBMEM is a powerful method to reduce noise in the original spectrum and to extract significantly reliable data.

\section{References}

[1] S. F. Gull and J. Skilling, "Quantified Maximum Entropy MemSys5 Users' Manual” ; Maximum Entropy Data Consultants Ltd., 1999.

[2] S. C. Lizamore, "Topics in Maximum Entropy Applications"; Master of Science in Statistics and Operations Research, Victoria Univ. of Wellington, 31 Aug. 1995.

[3] D. S. Sivia, "Bayesian Inductive Inference Maximum Entropy \& Neutron Scattering"; Los-Alamos Science Summer 1990.

a)

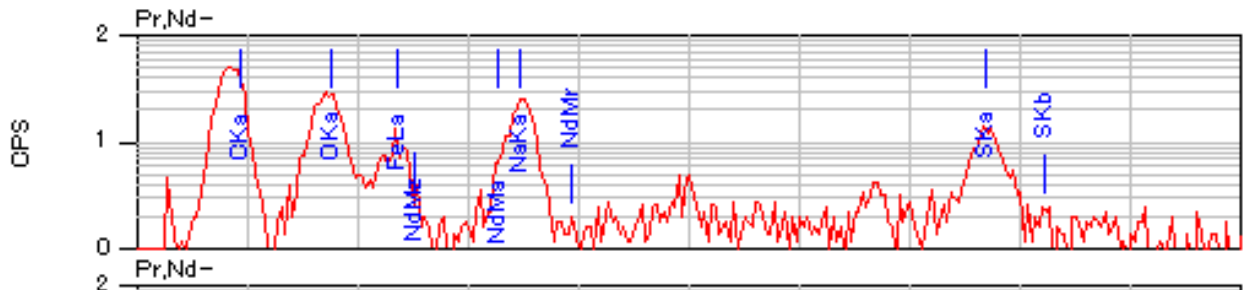

b)

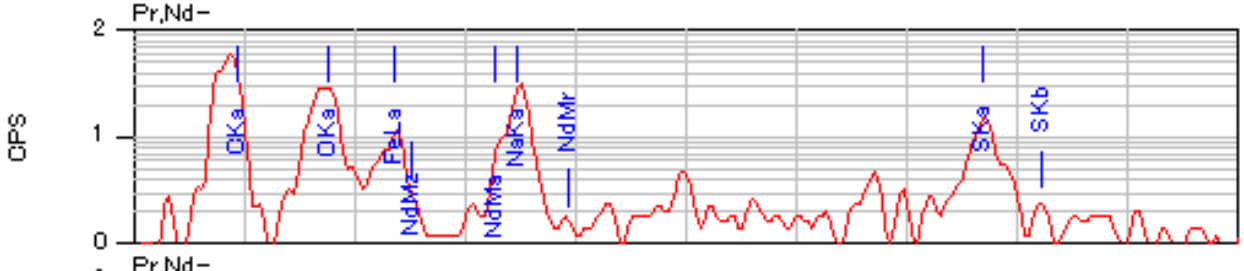

c)

d)
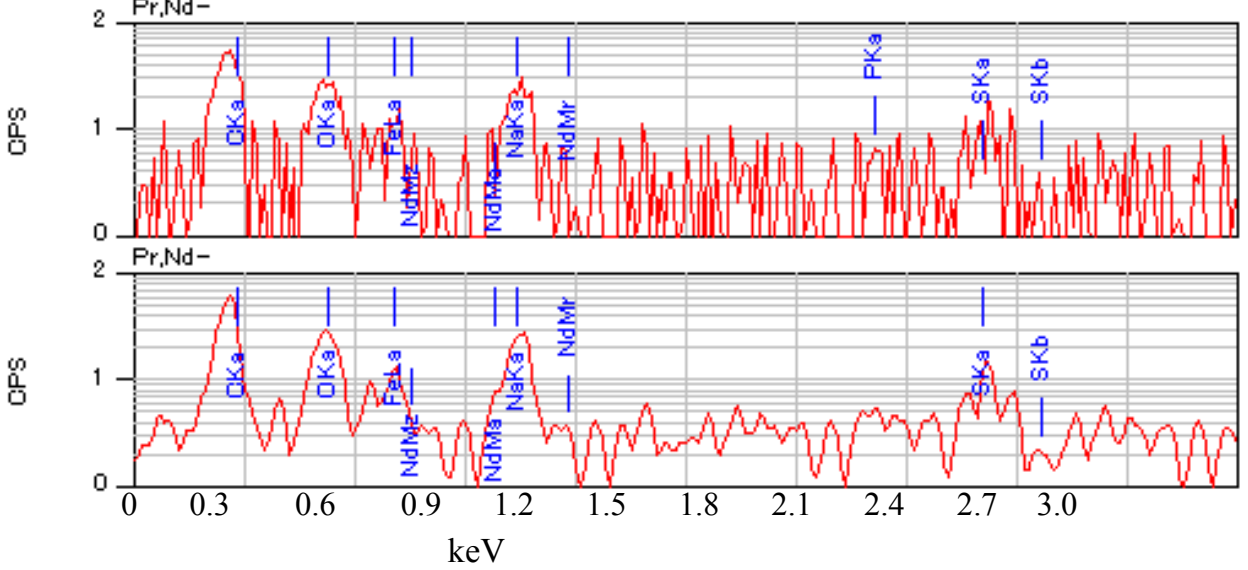
a) Original spectrum.
c) Artificial random noise is added to a)
b) Improved spectrum for a) by BBMEM.
d) Improved spectrum for c) by BBMEM.

Fig.1 EDX Spectrum of a specimen including rare metals

(Accelerating Voltage: $20 \mathrm{keV}$; Beam Current:0.605 nA; Sampling Time:5 s) 